\title{
ON SIGN LANGUAGE TOOLBOX AID
}

\author{
Mohanad ABDULHAMID \\ AL-Hikma University, Iraq \\ moh1hamid@yahoo.com
}

Ndiwa CHESEBE University of Nairobi, Kenya researcher12018@yahoo.com

\begin{abstract}
The world has been lately witnessing a landmark revolution in technology whereby more research and development is going into producing devices that are able to respond to human emotions, motions and behavior. These days, mobile phones are able to capture photos when the user is smiling or gesturing towards them. By making certain gestures, a user is able to control smart televisions and computers at the comfort of their homes, without the need of extra interface devices. The interaction between man and machines is being improved and made as natural as possible. More so, the application of hand gesture technology is finding use in making communication between people with hearing difficulties and those without, possible. This research presents the techniques that have made this possible. Most of these techniques however are in development stages. This is because of the significant challenges faced in providing this solution. First, there are over 30,000 sign language gestures which is quite a headache when trying to integrate them into a recognition system. Secondly, despite the fact that there are standard ways of making the sign language gestures, people have variations to them. Developers hence have to make allowance for these variations and in effect compromise on the accuracy of the system. This research evaluates the various algorithms developed, and tries to propose an algorithm that strikes the best balance between accuracy and allowance for variations in the sign language gestures.
\end{abstract}

KEYWORDS: sign language, gestures, toolbox aid

\section{Introduction}

According to statistics by the American Sign Language association, 90\% of deaf children are born in homes where the parents do not know or are not fluent in sign language. This provides a significant challenge since during early childhood, the interaction between the child and the environment is critical. As is the case in such scenarios, the children are taken to special needs schools that introduce them to sign language to help them express themselves (Wilbur \& Malaia, 2008).

However, as they grow, they are only able to communicate fully with people with the ability to use sign language. This means that they cannot converse properly with people who are not conversant with this language. More so, they are unable to use normal devices such as computers for their everyday duties (Efthimiou et al., 2009). 
Hence a need arises to try and bridge this gap. Currently, there are a number of gesture interfaces that allow a person to use sign language gestures which would then be translated to words, that another person could understand and conversely, another person types in words which are then converted to sign language gestures. There is no commercial sign language translator at the moment and these are just development ones (Dardas \& Georganas, 2011; Elmahgiubi, Ennajar, Drawil \& Elbuni, 2015).

However, despite there being progress in developing such interfaces, designing these systems is technically challenging due to the extremely wide sign language gesture vocabulary. There is a great need to have a proper and more accurate interface system since this will significantly improve communication between people with hearing difficulties and those without. This will also broaden the range of applicability in more natural user interface designs (Kumar, Thankachan \& Dominic, 2016).

This paper offers a solution in this modern world where every human aspect is being revolutionized by technology and computerized. Everywhere we go; there are numerous computers that we always interact with. However, it is rather discouraging that not so much emphasis has being put in researching and developing simple ways of communicating with the physically challenged, especially the deaf who are unable to communicate with people who are not conversant with sign languages. This proposal seeks to bridge this gap.

The algorithm developed in this paper strives to improve on the previous steps. It should be noted, that the significant contribution of this paper is that unlike previous works, this algorithm is able to detect the gestures of both hands simultaneously. This is a crucial consideration as it extends the capability of the other gesture detection systems.

\section{Design Methodology}

This section lays down the process followed in addressing hand gesture identification. The model of the solution is described and this is captured visually in the flow chart illustrated in Figure no. 1. The implementation of the identification process is divided into stages each of which is described in detail.

The first stage is image acquisition which details how a video stream obtained from the webcam and divided into frames which are then analyzed further on. Once the image is acquired, feature extraction is done whereby areas corresponding to the skin color of the hand. The image is further enhanced to remove unwanted areas to obtain a clean image. This is done through the use of a number of image processing tools. Finally, the fingers on both hands are identified and counted.

\subsection{Model of the Solution}

This paper is about creating an interface that allows the detection and counting of human fingers. This setup comprises of a computer webcam that acts as the input device, a MATLAB toolbox that processes the images captured by the webcam, isolates the hand gestures and indicates how many fingers are displayed on the screen.

The webcam is a video camera on the computer that feeds real time images to the computer for processing. The video feed consists of still images that are flipped at a rate of 30 images per second or 60 images per second. These still images are called frames. The captured images and videos are then made available to programs that want to use them. In this case we want to process the images in a MATLAB environment. Moreover, in this environment, there is an image processing toolbox that has the capability to support images and videos that have been captured by associated camera devices. By using a variety of functions, it is possible to use this toolbox to analyze and process captured devices according to the desires of the user. 
Once the program is able to track the fingers, a proper algorithm will be designed and implemented to enable us count the numbers of fingers that are displayed before the web camera. This means we will be able to count up to five fingers on one hand for both hands. Here, we will have to create a model for all the gestures that we want to be recognized. Depending on the algorithm developed, the determined number of fingers will be displayed on the screen to the user.

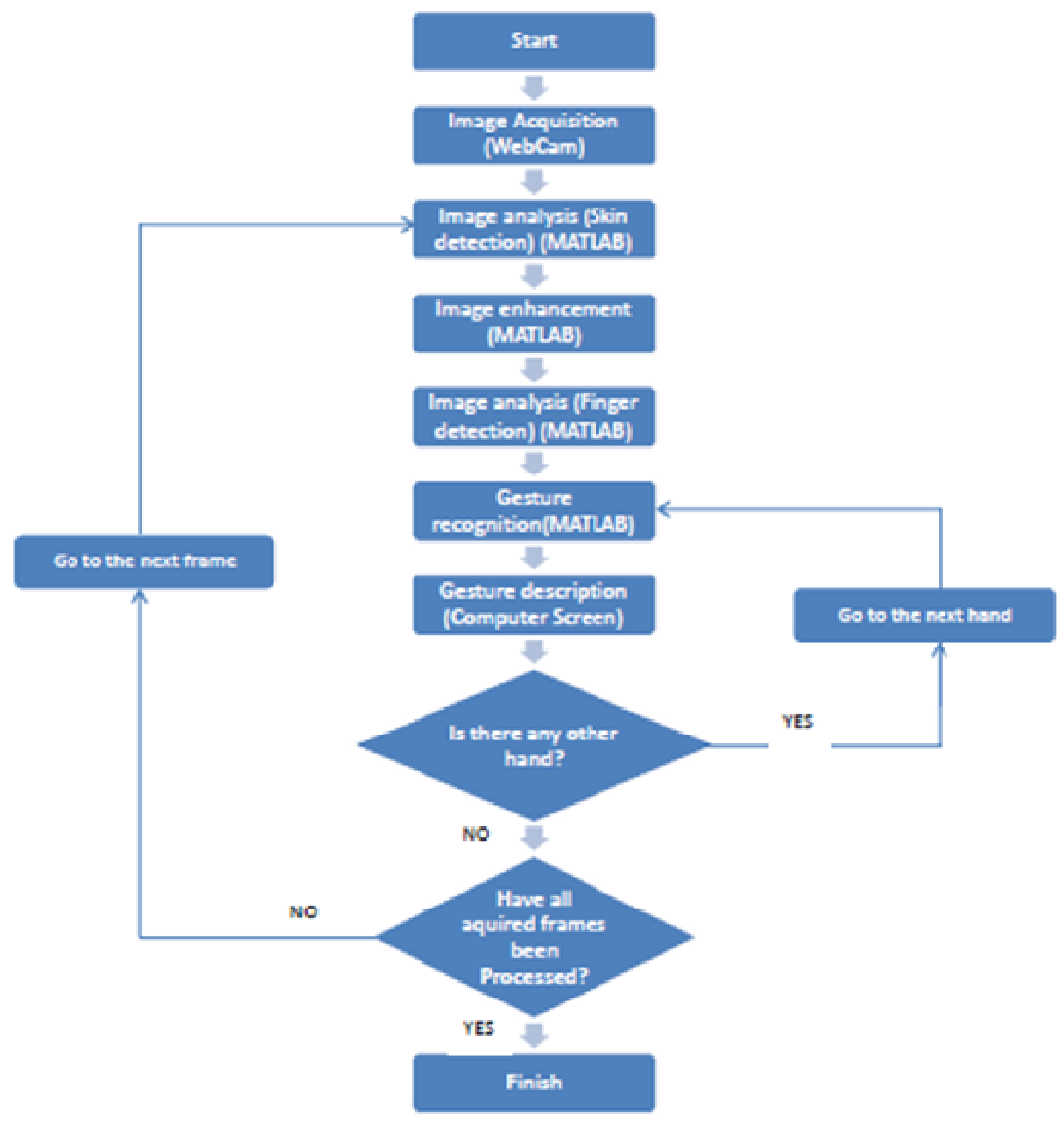

Figure no. 1: A model solution of the problem

\subsection{Implementation}

\subsubsection{Image Acquisition}

The initial step is to profile the 10 finger gestures for 10 people to represent the numbers $0-9$. This is done by taking the hand gestures using a smartphone camera (8 megapixels) then editing the images using the design software CorelDraw X7 to eliminate the background. Since this would be used to compare the real time images of the gestures taken by the webcam, it would be vital for them to be in high resolution. A sample set from one of the people profiled is shown in Figure no. 2. 


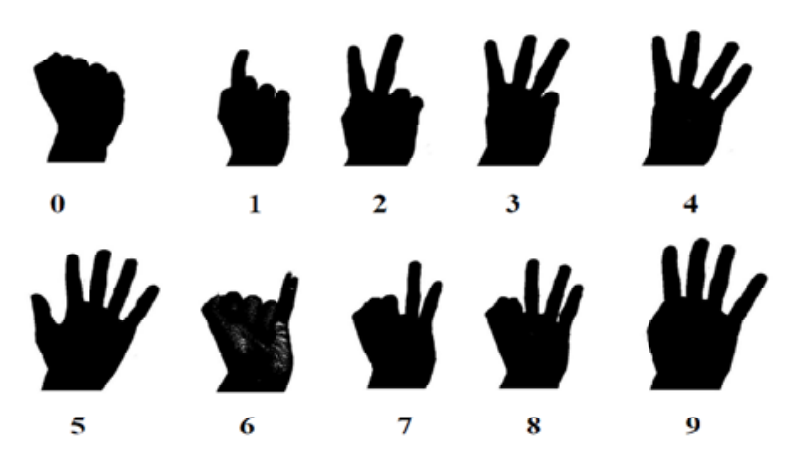

Figure no. 2: Images of the library of hand gestures to be used for comparison showing the number of fingers displayed

It should be noted that the images are of the left hand. This is so because the webcam inverts the orientation of images. Hence the hand that is desired to be captured is the right hand whose image will be inverted and compared with the profiled images above. The next step is to threshold the images such that they are in a black and white mode. The threshold image of the finger gesture representing 5 is shown in Figure no. 3.

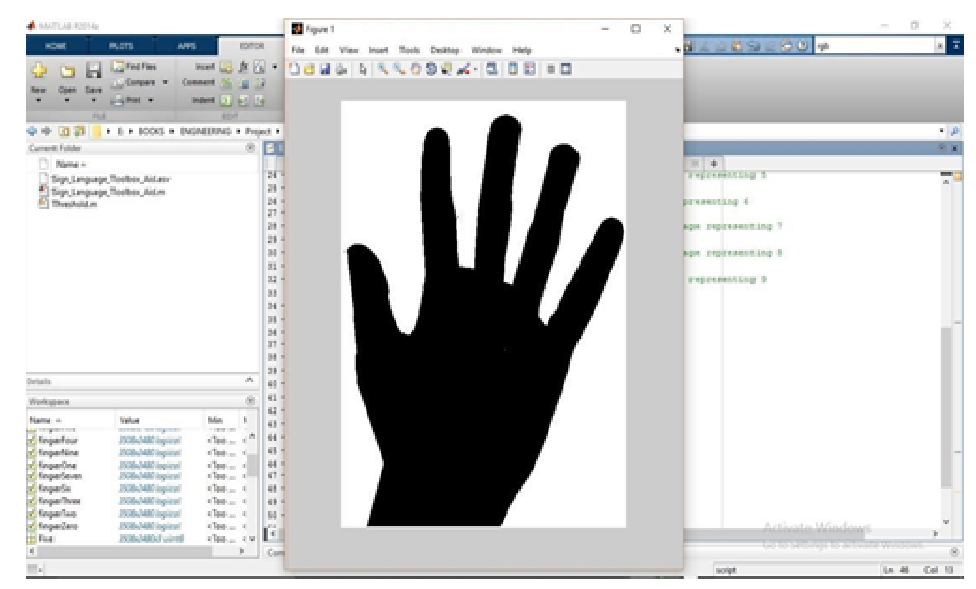

Figure no. 3: An image exposed to thresholding

Once the vocabulary of the fingers to be displayed is created, the next step is to build a graphical interface that allows a user to display fingers in front of the screen and at the same time view the output of the processing. This is done by using the Graphical User Interface Development Environment (GUIDE) tool in MATLAB which made this possible. The resultant user interface is as shown in Figure no. 4.

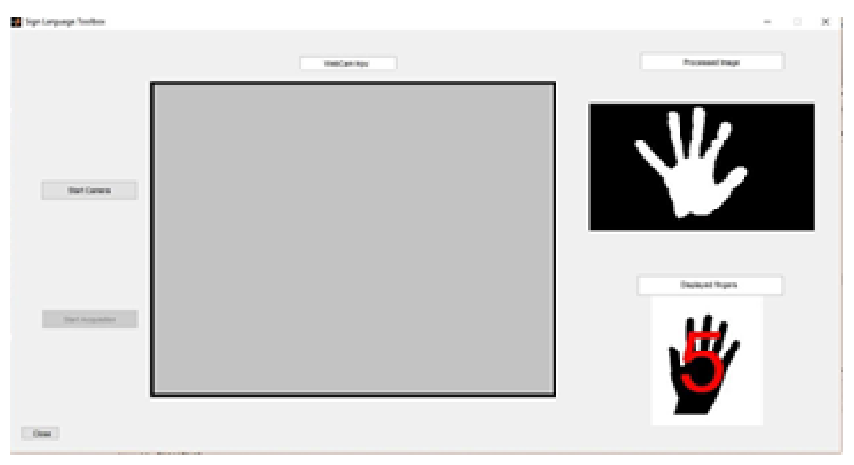

Figure no. 4: A preview of the graphical user interface 
On the graphical user interface, there are three areas that work to give the desired output. These are:

1. Webcam input area

2. Processed image area

3. Displayed fingers area

\subsubsection{Webcam Input Area}

In this area, the input from the computer webcam is streamed here. The video taken is used as the input which is to be processed in the system.

This area is under the control of two push buttons. The first one is the Start/Stop pushbutton which switches the camera on and off. The second pushbutton is the Start Acquisition/Stop Acquisition which when activated starts the image processing stage by acting as the trigger.

\subsubsection{Processed Image Area}

Once the start acquisition pushbutton is activated, the program takes one frame from the streaming video at a time, applies image enhancement changes on the image until the hands are clearly observed from the streaming video. The resultant processed image is then displayed in this area of the interface. Depending on how many frames have been triggered by the start acquisition pushbutton, this area will display all the frames in the processed format. In this case the frame rate is 30 frames per second which means if this rate is synchronized with the trigger; the output will be a processed video stream which will enable real time tracking and detection.

\subsubsection{Displayed Fingers Area}

Once the input video stream is reduced to a basic format where only the hands are visible, finger detection algorithms are applied on each frame and hence the fingers displayed will be counted and this outputted to the user interface in this area. The number changes real time as the detected fingers change.

\subsubsection{Image Analysis - Feature} Extraction

Once a single frame is grabbed from the video input, one feature of this image is identified and extracted so as to facilitate the gesture recognition. This feature is the skin color.

\subsubsection{Skin Detection}

In order to facilitate the process of gesture identification, an important step is to find a way to exclusively identify the palm of the hand from a mixed background of different objects. This unique identifier is the skin color. Moreover, as in most cases, the color of the palm and sole is much lighter than the rest of the human body. This makes recognition possible since regardless of the different skin color variations, the range of palm color for different people is narrower and hence can be captured for processing. The next step is finding a suitable color model to allow segmentation of the skin areas. These models and techniques are pixel-based as they classify each pixel in an image as skin region or non-skin region individually. Standard color imaging application models include RGB, HSV and YUV (YCrCb).

The RGB color model (see Figure no. 5) combines the three primary colors, Red, Yellow and Blue in different combination and superposition to form a certain color. Each of this color is called a component and its contribution value can be between fully on and fully off. These values are often represented as integers in the range of 0 to 255. Therefore a color in this model is described by the intensity of the three components. However, this model is not used in most image processing applications because it mixes hue, luminance and saturation together making it difficult to digitize images. 


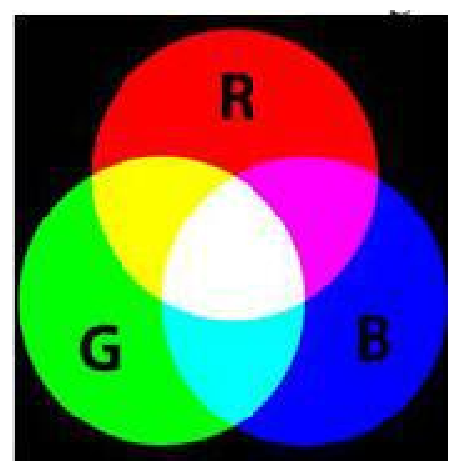

Figure no. 5: RGB color model

The HSV color model (see Figure no. 6) makes use of Hue (H), Saturation(S) and Value (V) to represent colors. Hue $[0,360]$ is expressed by angle, Saturations refers the distance to $\mathrm{V}$ axis and Value is simply luminance. This color space corresponds better to how people experience color compare with the RGB color space. Since by default the image captured by the webcam in the MATLAB environment is in RGB color space, conversion to the HSV color space is made.

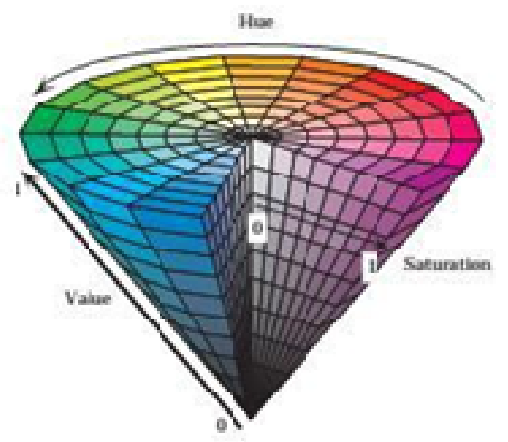

Figure no. 6: HSV color space

The YCbCr color model is commonly used in digital image and video processing. Here, the luminance data is represented by a component, Y, while, color data is represented by two color-difference components, $\mathrm{Cb}$ and $\mathrm{Cr}$. $\mathrm{Cb}$ is the difference between the blue component and a reference, while, $\mathrm{Cr}$ is the difference between the red component and a reference. Conversion of the default RGB to this color model is made possible.
In determining the most appropriate color space model for this paper, it is important to identify some basic performance limits of the selected color space. First, it is desired that the color space is not affected by luminance. Secondly, the selected color space had to have a good clustering feature.

Of the three, the $\mathrm{YCrCb}$ model offers a rigid solution because it separates the luminance component( $\mathrm{Y}$ ) from the chromatic component $(\mathrm{Cb}$ and $\mathrm{Cr})$. This makes this method have a higher discrimination between skin pixels and nonpixel areas over varying illumination conditions and therefore effective in characterizing various human skin colours.

From studies, it has been shown that pixels from skin regions are characterized by similar chrominance $(\mathrm{Cb}$ and $\mathrm{Cr}$ ) and cover all human races. It has also been shown that even with varying intensity of luminance; the skin color distribution in the $\mathrm{Cb}-\mathrm{Cr}$ subspace is not altered. This is because luminance is simply the brightness of a chromatic value. However, despite efforts to make this model $100 \%$ efficient, there exists possibility of extracting non-skin regions which have a similarity in color composition to the skin. This is referred to as false alarms. The standard ranges of values for the three components are as given below:

$$
\begin{aligned}
& \mathrm{Y}>80 \\
& 85<\mathrm{Cb}<135 \\
& 135<\mathrm{Cr}<180 \\
& \text { And } \mathrm{Y}, \mathrm{Cb}, \mathrm{Cr}=[0,255]
\end{aligned}
$$

However, in order to capture skin regions more accurately, a proposed range for the $\mathrm{Cb}$ and $\mathrm{Cr}$ components is as follows:

$$
\begin{aligned}
& 80<\mathrm{Cb}<120 \\
& 133<\mathrm{Cr}<173
\end{aligned}
$$


The next step is to create a test data from which more detailed analysis is to be done. This involves collection of hand images from people of different ages, descent, sex and size in order to create a compromise reference. The test data also involves testing the effect of different lighting conditions on the hands.

\subsubsection{Image Enhancement}

When the web camera starts to record, the desire is to process the frames until the only visible object is the fingers for the ease of detection. This is critical since most of the time the background could have many objects which may make it impossible to detect the hands. Therefore a number of enhancement techniques have to be used together to make this possible.

It is observed that the image enhancing tools clean up the image frame such that only the hand is possible. The above demonstration is a worst-case scenario where numerous objects exist in the background of the frame. The performance of this image processing stage is tested and proven that good lighting plays a major role in the tracking of the hand. Moreover, having a background of a uniform color (preferably white) improves the processing incredibly. In this case the following image enhancement tools are used to achieve the processed images.

\subsubsection{Gray Scale Conversion (rgb2gray)}

This tool converts an image from its true color image which is in the RGB format to the gray scale intensity format by removing the hue and saturation information in the image but retaining the luminance. A gray scale image is a data matrix whose values represent shades of the color gray.

\subsubsection{Black/White Conversion (im2bw) \\ After the image has been transformed into the gray scale format, this tool further}

converts the resultant image to a binary image where the pixels that have a luminance greater than a certain value are given a value 1 and replaced with black color, while, the pixels that have a luminance value less than that value are given the value 0 and replaced with the color white. A binary image is also described as a logical array of zeros and ones. This process is called thresholding.

\subsubsection{Black/White Areas Filter} (bwareaopen)

This function works on the resultant image that has been converted into binary. Here, the connected areas on the binary image that have fewer than a certain number of pixels are removed from this binary image. This is to ensure specks of unwanted objects are filtered out of the image and hence clean up the image. The operation is known as black and white area opening. The resulting image is a cleaned up binary image.

\subsubsection{Flood Fill (imfill)}

This operation changes the connected pixels in the background (0s) to foreground pixels (1s) until it reaches the image object boundary. After the unwanted spots are removed from the binary image, holes are left on the image and it is necessary to fill them up with the colors in the adjacent neighborhood. This function implements that action to ensure a smooth and clean image.

\subsubsection{Binary Erosion (imerod)}

Erosion is a fundamental morphological operation that removes pixel on object boundaries. The output pixel has a value that is the minimum of the pixels that are in the neighborhood of the input pixel. If any of these pixels is set to the value 0 , then the output pixel is set to 0 . This operation erodes the binary image to further clean up the image. It has the effect of eliminating noise and filling holes inside images. 


\subsubsection{Binary Dilation (Imdilate)}

In line with the binary erosion function above, this function dilates the binary image that has been eroded. Dilation is a fundamental morphological operation that adds pixels to the boundary of an object in an image. The output pixel has a value that is the maximum of the pixels that are in the neighborhood of the input pixel. If any of these pixels is set to the value 1, then the output pixel is set to 1 . These two morphological processes (dilation and erosion) are often used together to implement image processing operations.

\subsubsection{Median Filtering}

The operation sets the input pixels of an image to the value of pixels in the neighborhood of the particular pixels. The value of the output pixel is determined by the median of the pixels in the neighborhood unlike other filtering methods that use the mean. This function performs median filtering of the image in two dimensions and in doing so, removes the imperfections that result from the combination of image processing tools above. A sample of this combination is shown in Figure no. 7.
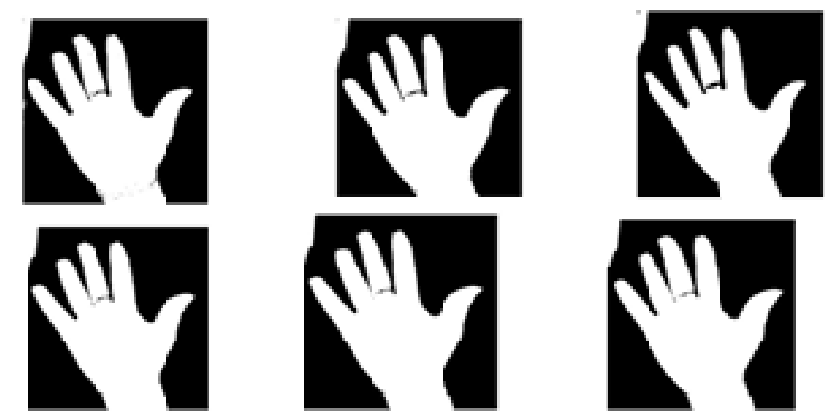

Figure no. 7: The six stages applied in the Image enhancement process in the order

As a result of the above processes, the image captured in the frames is reduced to a format that makes it possible to extract features from the video frames that will allow figure gesture identification.

\subsubsection{Gesture Identification}

Once the palm of the hand is extracted from the image, the next step is to establish a way to locate fingers from this image of the palm. First, the captured hand is in binary format, isolated from the background and hence the MATLAB workspace treats it as an object. The centroid of this hand object is obtained by using inbuilt functions. This centroid is the most central point of the hand object will be the reference from which the identification process will be built upon.

From this processed binary image, a contour can be traced in the boundary between the black and white areas.
The pixels that lie on this contour are analyzed to find which of them form the maximum data points relative to a reference point which in this case is the centroid of the hand object. These will become the peaks of the object. This is done by taking each pixel on the contour, measuring its distance from the centroid and comparing this value with the values of the pixels in the neighborhood. Therefore, the pixels that are farthest from the centroid compared with the adjacent pixels will be marked as peaks and the value of total peaks stored.

This is done so because the fingertips are the furthermost part on human hands and clearly locating these points will enable the identification of the fingers and hence make it possible to count them. As expected, this process gives us peaks that might not necessarily be the fingertips and therefore appropriate tuning processes are required to improve the accuracy. 
The first filter process is to consider only the peaks that are a certain distance away from the centroid and filter out those that are not. This minimum distance is determined by the least expected value of the peak which should be the thumb. Any peak below this value is ignored. The next filter process is to get the distance between adjacent peaks and setting a minimum value so that not more than one peak can be obtained from the one finger. This minimum value is determined by the average minimum distance between fingers. The combination of these two filters improves the performance of the fingertips identification significantly and hence is able to count fingers that are displayed.

Once the peaks have been positively identified, the next stage is to mark them so that they can be tracked. This is simply drawing markers of any size and color on the pixels that form the peaks. The number of peaks is retrieved and displayed. This constitutes the identification and counting of fingers in one frame. By looping, the process is repeated for multiple loops and hence real time tracking and counting of fingers.

\section{Results}

After the gesture algorithm is completed, it is tested under different
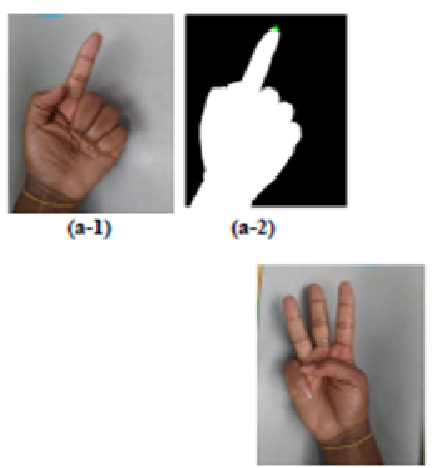

(c-1)

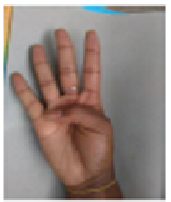

(d-1)

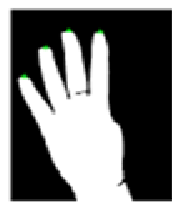

(d-2) conditions in order to see the performance. Fifteen people volunteered to have images of their hands taken to create a data set that would work for all skin colors. The demography of this sample included people of different skin color, age, sex and race. The images of these hands are processed by the same algorithm which identified the peaks from them. The peaks are marked by the green marker, and the number of peaks is given by the number of the markers. The testing conditions are classified under these categories:

1. Under ideal conditions

2. Varying lighting conditions

3. Extreme lighting conditions

4. Varying skin colors

5. Complex backgrounds.

Under each category, the results are displayed, discussed and explained.

\subsection{Under Ideal Conditions}

Here, the ideal conditions for testing are utilized. These ideal conditions are proper lighting and a uniform background. As shown in Figure no. 8, Figure no. 9, and Figure no. 10, the accuracy by which the algorithm detected the fingers is optimized and the results satisfying.
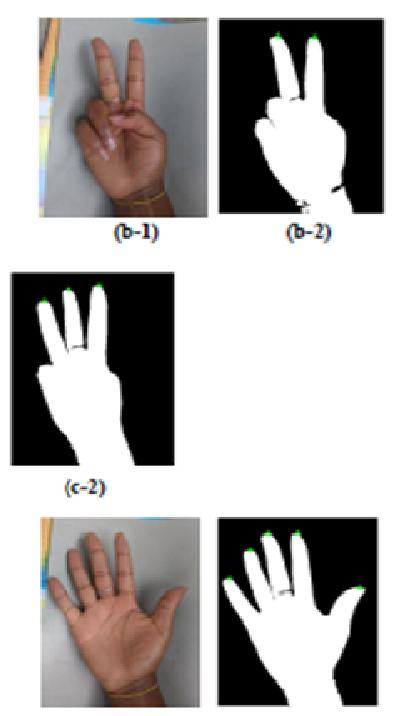

(e-1)

Figure no. 8: Images showing identified fingers from data set 1 with light uniform background 


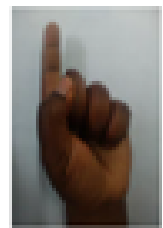

(1-I)

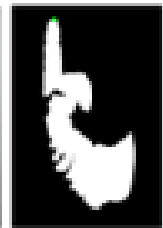

(4)

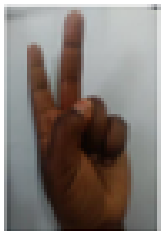

(b-1)

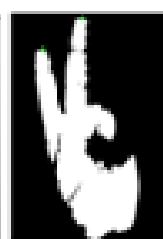

(1)-2)
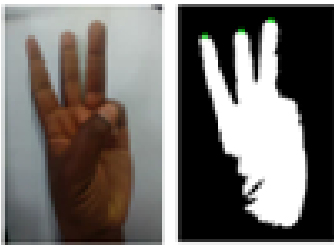

$(6-1)$

$(a-1)$

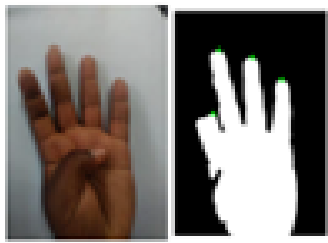

$(4-1)$

(d-1)

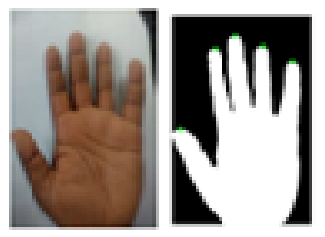

$(4-1)$

(a)

Figure no. 9: Images showing identified fingers from data set 2 with light uniform background

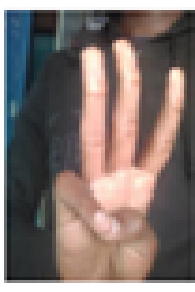

(1)-1)

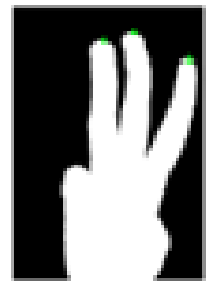

(1-1)

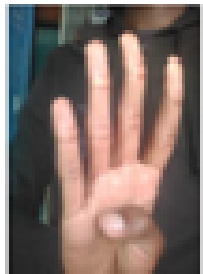

0-1)

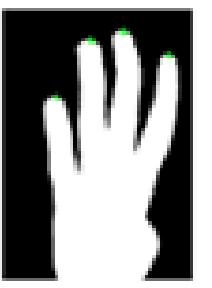

(b-i)

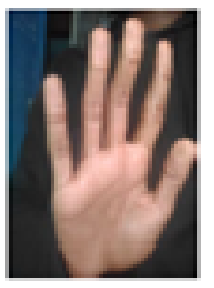

(c-1)

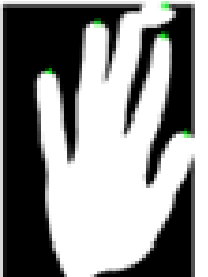

$(0-1)$

Figure no. 10: Images showing identified fingers from data set 3 with dark background

\subsection{Under Varying Lighting} Conditions

In this category, the luminance of the environment is varied while the background is maintained at a uniform color. The palm of the hand is glossy and reflective hence the color changes rapidly according to the changes in lighting. The results shown in Figure no. 11 are satisfying as the algorithm is able to pick out the fingers from the images despite the variance in lighting. 


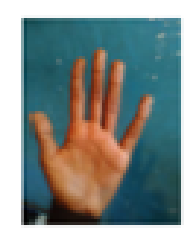

(1-1)

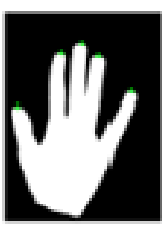

$(1+\cdots)$

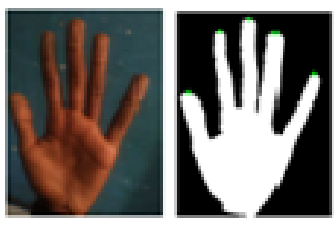

(b-1)

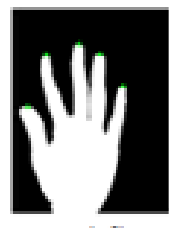

$(-2)$

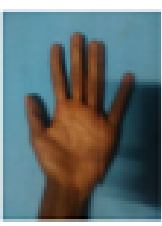

(4-1)

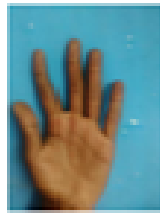

(6-1)

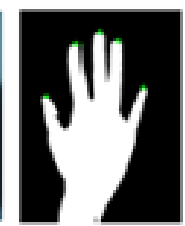

[-1]

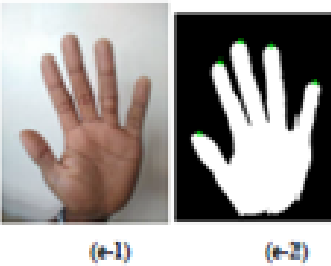

Figure no. 11: Images showing identified fingers from data set 4 with varying lighting conditions on uniform background

\subsection{Under Extreme Lighting Conditions \\ Under extreme lighting conditions, the algorithm was unable to pick out the fingers from the images. As shown in Figure no. 12, in very dark conditions (b, c, $\mathrm{d}$ and $\mathrm{e}$ ), image processing tool is unable to detect the skin color since none of the constituent colors in the images falls above}

the minimum threshold required to classify it as a skin region, hence these images give negative results. In very bright conditions (a), the glossy nature of the palm reflects much of the light, making the palm brighter and hence this surpasses the maximum threshold required to classify this as a skin region. This image therefore returns negative results.

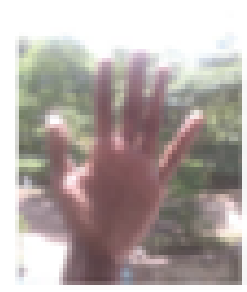

(1)

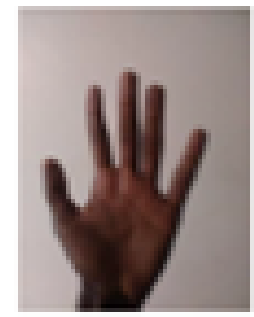

(b)

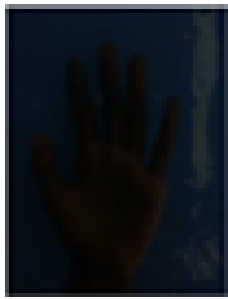

(c)

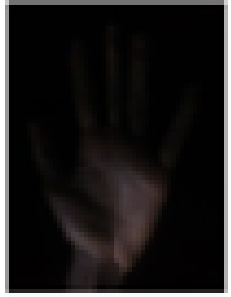

(4)

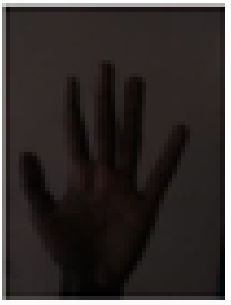

(i)

Figure no. 12: Images showing identified fingers from data set 5 with extreme lighting conditions

\subsection{Varying Skin Colors}

Given that human have different skin colors, it is necessary to test various skin colors and see if the color space range that is chosen factored all of these variations. The algorithm is able to positively identify the skin regions but also processed false alarms, which are areas that are non-skin but have the same color as the skin regions. This results in fairly distorted processed images as shown in Figure no. 13. 


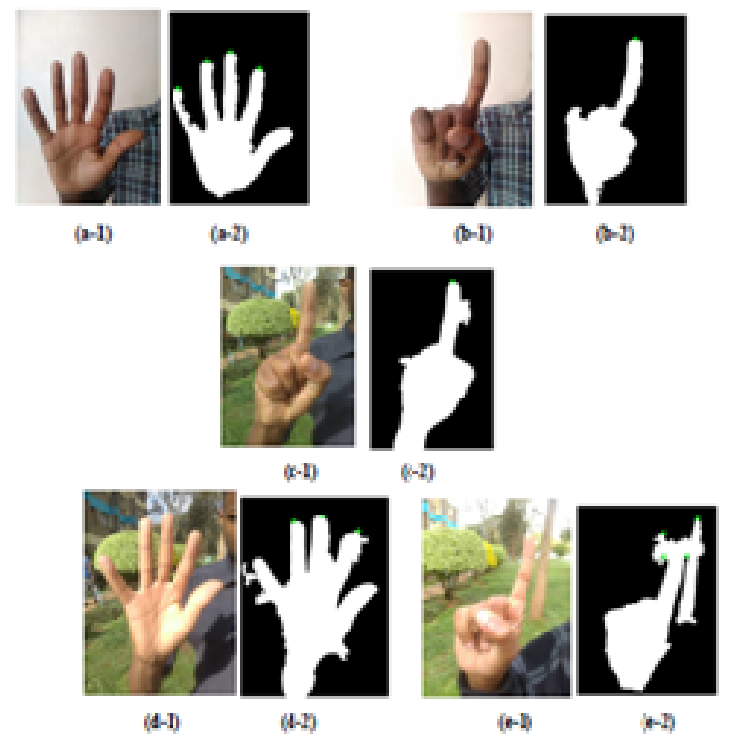

Figure no. 13: Images showing identified fingers from data set 6 with varying skin color

\subsection{Complex Background}

In this category and as shown in Figure no. 14, images that are taken in environments where the background contained false alarms are considered. These false alarms are processed by the algorithm as skin areas when they are not hence give false results. This is not easily rectifiable because narrowing the range for skin regions will reduce the variety of skin colors that can be positively identified. The only solution as seen in the previous results and earlier mentioned would be limiting the environment for gesture identification to a uniform background, preferably white.

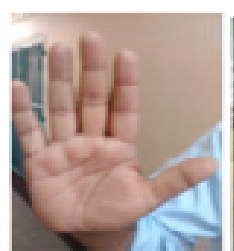

(s)

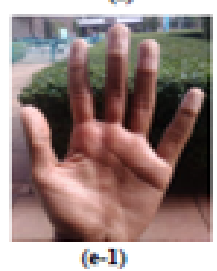

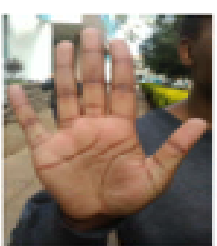

(b)

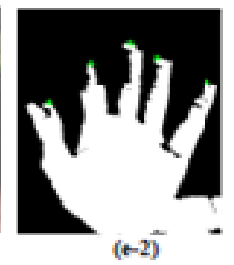

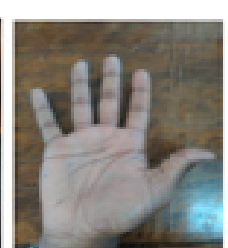

(c)

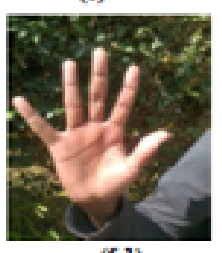

(6-1)

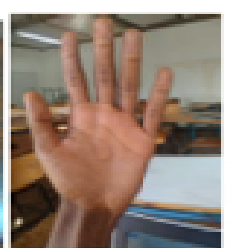

(d)

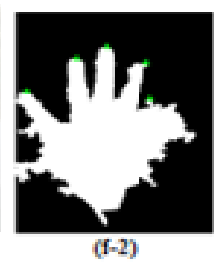

Figure no. 14: Images showing identified fingers from data set 7 with complex backgrounds

\subsection{Implementation of Two Hand}

\section{Detection and Recognition}

Once the algorithm works satisfactorily for one hand, a loop is introduced such that the detection function is always listening for any other object that corresponded to the shape of the hand. Once this other hand is detected, the counting operation shifted to this new hand and the number of fingers displayed. The algorithm would then use this as a basis and listen for any other hand and the process continues. This results in a system that counted the fingers on both hands as shown in Figure no. 15. 


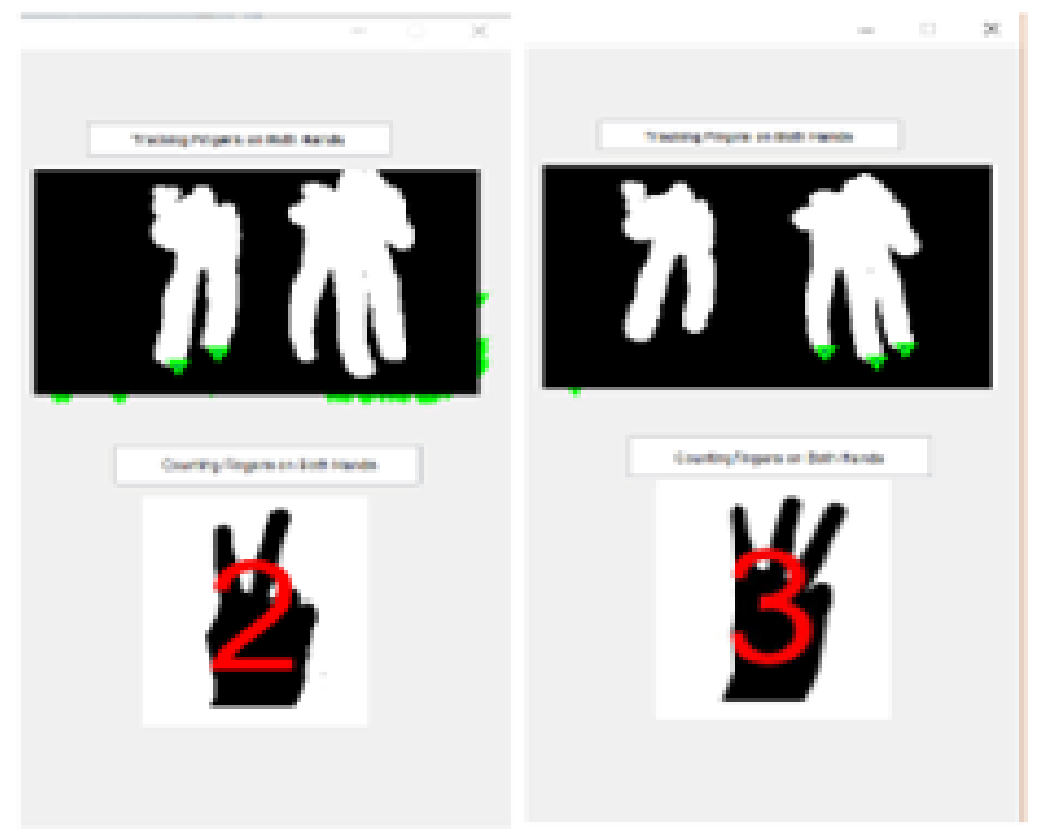

Figure no. 15: Two hand detection and recognition

\section{Conclusion}

An image processing algorithm for real time hand gesture recognition was designed, implemented and tested. This algorithm was able to track and count human fingers on both hands across various backgrounds in real time. This system provided a graphical interface that made it simple for the end user to interact with it. Moreover, only a camera and a computer were used as the only components and facilities hence this makes it easy to integrate the system with other existing systems to extend their capability.

As tested, the system has a high accuracy and was able of detect the hands of about $90 \%$ of the tested subjects. The limitation for achieving full efficiency was the lighting conditions which is an important factor affecting image processing. An important aspect of this system is that it is able to count the fingers of most people even with different hand geometries. This was observed during testing whereby some people had hands that had fingers - to - palm ratios beyond the normal ratios. This concern was solved by having the reference to which the finger counting was based to vary according to the user.

Such a system would be useful in schools for the deaf, where the children with hearing difficulty could find it possible to communicate with those children without hearing impairment. Since this system is reversible, such that numbers can be translated to hand gestures, the users without hearing problems can send a message to them flawlessly. Websites plugins could implement such a system to allow these deaf children interact with the internet by accepting their hand gesture inputs and translate this to words or actions.

This system could also find its use in scenarios where a hand signal is needed to control a certain action such as switching the lights in a room or closing a door. 


\section{REFERENCES}

Dardas, N., \& Georganas, N. (2011). Real-time hand gesture detection and recognition using bag-of-features and support vector machine techniques. IEEE Transactions on Instrumentation and Measurement, Vol. 60, Issue 11, 3592-3607.

Efthimiou, E., Fotinea, S.-E., Hanke, T., Vogler, Ch., Glauert, J., Bowden, R., Braffort, A., Collet, Ch., Maragos, P., \& Segouat, J. (2009). Sign language recognition, generation, and modeling: A research effort with applications in deaf communication, Universal Access in Human-Computer Interaction. Addressing Diversity: 5th International Conference, (UAHCI), Held as Part of HCI International 2009, San Diego, CA, USA, Proceedings, Part I, 21-30.

Elmahgiubi, M., Ennajar, M., Drawil, N. M., \& Elbuni, M.S. (2015). Sign language translator and gesture recognition. IEEE Global Summit on Computer \& Information Technology, Tunisia.

Kumar, A., Thankachan, K., \& Dominic, M. (2016). Sign language recognition. IEEE $3^{\text {rd }}$ International Conference on Recent Advances in Information Technology, India.

Wilbur, R.B., \& Malaia, E. (2008). Contributions of sign language research to gesture understanding: What can multimodal computational systems learn from sign language research. International Journal of Semantic Computing, Vol. 2, Issue 1, 5-19. 